\title{
Lesson of the month 1: To stop a fit, but swinging low
}

\author{
Authors: Osakpolor Ogbebor, ${ }^{A}$ Ankit Agrawal ${ }^{B}$ and Balaji Yegneswaran ${ }^{C}$
}

This is a case of an elderly woman who presented to our emergency room with an episode of a witnessed fall. The past medical history of the patient was significant for post-stroke epilepsy for which she was on oxcarbazepine. Initial blood work showed a white cell count of 4.5 , haemoglobin of 12.4 , and platelet count of 15,000 . Peripheral blood smear showed normal platelet and red cell morphology without clumping. The patient's history suggested that she was recently started on oxcarbazepine prompting discontinuing of the drug. The platelet count improved from 15,000 cells $/ \mathrm{mL}$ to 80,000 cells $/ \mathrm{mL}$ on discharge.

Antiepileptic medications have been reported to cause various blood dyscrasias in the literature. There are few studies that report the association of carbamazepine and thrombocytopenia and much fewer written about oxcarbazepine. Thrombocytopenia appears to be an uncommon reported side effect of oxcarbazepine; more commonly reported side effects include dizziness, tiredness, memory problems and headache. The treatment of antiepileptic drug-associated thrombocytopenia is discontinuing the medication and monitoring the platelet counts. In few cases, immunoglobulin infusion is required. Antiepileptic drug-associated thrombocytopenia is difficult to predict and so it is imperative to monitor the platelet level when antiepileptic drugs are started and even after the medication is switched to a different one.

KEYWORDS: Oxcarbazepine, carbamazepine, thrombocytopenia, antiepileptic drugs, side effect

\section{Background}

Antiepileptic drugs (AEDs) are commonly used in the management of seizure disorders. However, they are associated with several side effects such as nausea and vomiting, hyponatremia, dizziness, fatigue and elevated transaminase - which are common - as well as some rare side effects such as hypersensitivity reaction, blood dyscrasia, porphyria and aseptic meningitis. Management of seizure disorders is difficult as physicians must balance the need for compliance with the development of adverse effects. Oxcarbazepine is an analog of carbamazepine and is reported to

Authors: A resident physician, Rutgers / Saint Peter's University Hospital, New Jersey, USA; ${ }^{B}$ resident physician, Rutgers / Saint Peter's University Hospital, New Jersey, USA; ' attending physician, Rutgers / Saint Peter's University Hospital, New Jersey, USA be better tolerated. Here we report a case of a rare side effect of this medication.

We searched databases (Medline, EBSCOhost, Cochrane library) with the following search terms: oxcarbazepine, carbamazepine, platelets, thrombocytopenia, blood dyscrasia, adverse effects. Studies were reviewed and additional references were explored.

\section{Case presentation}

This is a case of an elderly female who presented to our emergency room with an episode of a witnessed fall which occurred while she was trying to get out of the bed. Prior to the fall, she did not report any changes in vision, smell or taste, spinning sensation of the room, palpitations or chest pain. Following the fall, there was no loss of consciousness, abnormal body movement, incontinence, tongue biting, trauma to the head or confusion. She reported being fatigued and having intermittent headache for a couple of weeks prior to presentation. Review of other systems was unremarkable and the patient was negative for fever.

The past medical history of the patient was significant for irritable bowel syndrome, chronic obstructive pulmonary disease and haemorrhagic stroke which occurred a few years previously. She developed post-stroke epilepsy in the next year for which she was on an anti-epileptic drug. The patient had been on levetiracetam ever since her first episode of seizure but was recently changed to oxcarbazepine because of fatigue 3 weeks before presenting to the emergency room.

Vitals on presentation were: blood pressure $135 / 64 \mathrm{mmHg}$ without orthostatic hypotension; pulse 86 beats per minute, regular rhythm and good volume; respiratory rate 16 breaths per minute; temperature $36.8^{\circ} \mathrm{C}$ and oxygen saturation was $96 \%$ on room air. Physical examination was within normal limits.

\section{Investigations}

Initial blood work showed a white cell count (WCC) of 4.5 cells / cubic millimeter, haemoglobin of $12.4 \mathrm{~g} / \mathrm{dL}$, and platelet count of 15,000 cells $/ \mathrm{mL}$. Prothrombin time was 12.1 seconds and partial thromboplastin time (PTT) was 25.6 seconds. The chemistry panel was normal with a sodium level of $140 \mathrm{mEq} / \mathrm{L}$, blood urea nitrogen $18 \mathrm{mg} / \mathrm{dL}$ and creatinine of $0.84 \mathrm{mg} / \mathrm{dL}$. Peripheral blood smear showed normal platelet and red cell morphology without clumping. On reviewing her records from the neurology clinic 3 months prior to presenting, her complete blood count (CBC) was within normal limits. Computed tomography of the head without contrast showed encephalomalacia of the right frontal and anterior temporal lobes secondary to prior parenchymal haemorrhages with no acute findings. 


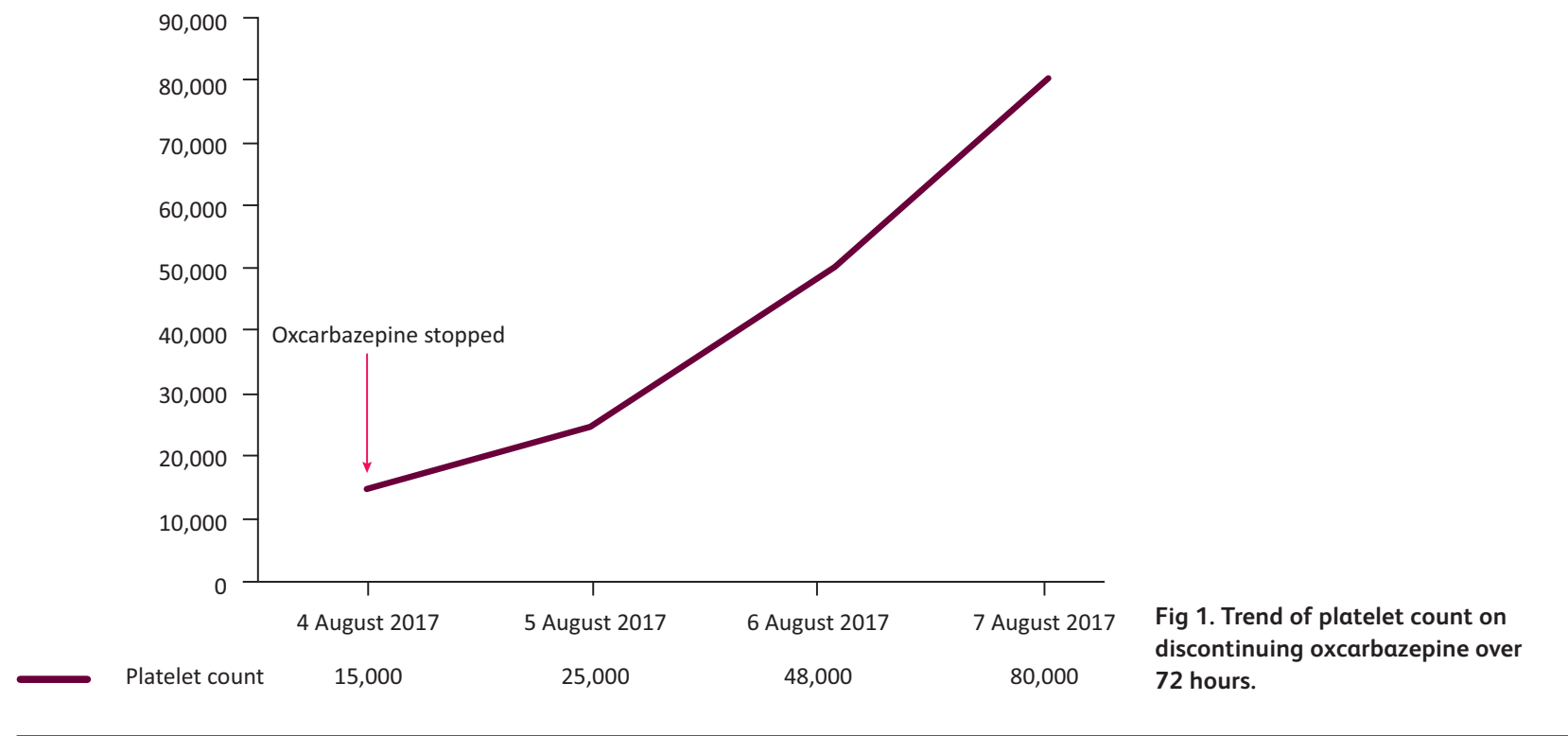

\section{Treatment}

Based on the history and the initial blood work and peripheral blood smear, which showed no coagulopathy, a normal renal function and a blood film with normal cell morphology, a diagnosis of drug-induced thrombocytopenia was considered. As the history suggested that the patient had recently started oxcarbazepine, the drug was discontinued. Platelet count improved from 15,000 cells/ $\mathrm{mL}$ on presentation to 80,000 cells $/ \mathrm{mL}$ on discharge with no bleeding manifestations (Fig 1). She was haemodynamically stable throughout the hospital stay. Neurology was also consulted and she was discharged on lamotrigine. Of note, the patient reported her headache resolved prior to discharge.

\section{Discussion}

On encountering thrombocytopenia, defined as a platelet count less than 150,000 cells $/ \mathrm{mL}$, one should initially rule out pseudothrombocytopenia, which is caused by clumping, by reviewing a peripheral blood smear. The differential diagnosis of 'true' thrombocytopenia includes infection-induced thrombocytopenia, disseminated intravascular coagulopathy (DIC), immune thrombocytopenic purpura (TIP), thrombotic thrombocytopenic purpura and drug-induced thrombocytopenia.

Antiepileptic medications have been reported in the literature to cause various blood dyscrasias. ${ }^{1}$ This includes an extensive list of medications consisting of levetiracetam, gabapentin, phenytoin, sodium valproate, lamotrigine and carbamazepine. There are few studies that report the association of carbamazepine and thrombocytopenia and much fewer written about oxcarbazepine.

Oxcarbazepine was created by modifying the structure of carbamazepine. $^{2}$ The aim was to reduce the production of toxic metabolites of carbamazepine following degradation by the hepatic cytochrome P-450 enzyme pathway. It is not metabolised by the liver and has less drug-drug interaction such as with the oral contraceptive pill and other AED. Mechanism of action is similar as they both block calcium and sodium channels; however, a different type of calcium channel is blocked by oxcarbazepine. ${ }^{3}$
It has been observed that there is a reduction in side effects such as rash, thyroid disease and sexual disorders with oxcarbazepine and it was better tolerated. However, more cases of hyponatremia were seen with oxcarbazepine.

The mechanism of carbamazepine-induced thrombocytopenia is not clearly understood. It is postulated that it could be immune mediated or by direct toxicity. In a study that used a challenge test after a previous exposure, platelet associated $\mathrm{IgG}$ and interleukin 6 were elevated indicating an immune mediated pathway. ${ }^{4}$ It has also been theorised that carbamazepine, or its metabolites, exerts a direct toxic effect on myeloid cells and through this mechanism causes aplastic anaemia. ${ }^{5}$ In cases of other AEDS, there appears to be idiosyncratic synergic reaction when an AED is combined with either glucocorticoids, a proton-pump inhibitor, $\mathrm{H} 2$ antagonist or another AED. ${ }^{6}$ Oxcarbazepine is not metabolised by the liver; therefore, one may suspect that its side effects may not be mediated directly by toxic metabolites. Oxcarbazepine has also been shown to cause haemolytic anemia by an unknown mechanism. ${ }^{7}$

Nevertheless, thrombocytopenia appears to be an uncommon reported side effect of oxcarbazepine. In a review of 161 patients in an outpatient clinic in London, ${ }^{8}$ the commonest reported side effects were dizziness, tiredness and memory problems. It also causes severe headache ${ }^{9}$ which our patient reported as one of her symptoms.

The treatment of AED-associated thrombocytopenia remains the same: with discontinuing the medication there is remarkable improvement in platelet counts. In few cases, intravenous immunoglobulin was required. AED-associated thrombocytopenia is difficult to predict and often at times there is the need to continue some form of anti-seizure drug in the patient involved. Therefore, it is imperative to monitor the platelet level even after the medication is switched to a different one.

\section{References}

1 Blackburn SCF, Oliart AD, Rodríguez LAG, Gutthann SP. Antiepileptics and blood dyscrasias: A cohort study. Pharmacotherapy 1998;18:1277-83. 
2 Schmidt D, Elger CE. What is the evidence that oxcarbazepine and carbamazepine are distinctly different antiepileptic drugs? Epilepsy Behav 2004;5:627-35.

3 Millichap JG. Risk-benefits of oxcarbazepine vs carbamazepine. Pediatric Neurology Briefs 2004;18:78-9.

4 Ishikita T, Ishiguro A, Fujisawa K, Tsukimoto I, Shimbo T. Carbamazepine-induced thrombocytopenia defined by a challenge test. Am J Hematol 1999;62:52-5.

5 Rao KV. Drug-Induced Hematologic Disorders. In: DiPiro JT, Talbert RL, Yee GC et al (eds), Pharmacotherapy: A pathophysiologic approach, 9th edn. New York, NY: McGraw-Hill, 2014.

6 Ranzino AM, Sorrells KR, Manor SM. Possible acute thrombocytopenia post esomeprazole and hydantoin coadministration. J Pharm Pract 2010:23:140-3
7 Chaudhry M, Abrar M, Mutahir K, Mendoza C. Oxcarbazepine-induced hemolytic anemia in a geriatric patient. Am J Ther 2008;15:187-9.

8 Besi E, Boniface DR, Cregg R, Zakrzewska JM. Comparison of tolerability and adverse symptoms in oxcarbazepine and carbamazepine in the treatment of trigeminal neuralgia and neuralgiform headaches using the Liverpool Adverse Events Profile (AEP). J Headache Pain 2015;16:1-7.

9 Piovesan E, Teive H, de Paola L et al. Uncontrolled headache induced by oxcarbazepine. J Headache Pain 2007;8:301-5.

Address for correspondence: Dr Osakpolor Ogbebor, Saint Peter's University Hospital, 240 Easton Avenue, New Brunswick, New Jersey 08901, USA Email: osakpolor.ogbebor@yahoo.com 